\title{
Contact force and impedance decrease during ablation depends on catheter location and orientation: insights from pulmonary vein isolation using a contact force-sensing catheter
}

\author{
Sven Knecht ${ }^{1,2} \cdot$ Tobias Reichlin $^{1,2} \cdot$ Nikola Pavlovic $^{1} \cdot$ Beat Schaer $^{1,2}$. \\ Stefan Osswald $^{1,2} \cdot$ Christian Sticherling ${ }^{1,2} \cdot$ Michael Kühne $^{1,2}$
}

Received: 6 October 2014 / Accepted: 30 March 2015 /Published online: 30 April 2015

(C) Springer Science+Business Media New York 2015

\begin{abstract}
Purpose Contact force (CF) sensing during radiofrequency (RF) ablation allows controlling lesion size. The aim of this study was to analyze the impact of catheter tip location and orientation on the association of $\mathrm{CF}$ and impedance decrease. Methods We retrospectively analyzed RF applications from 32 patients undergoing catheter ablation for paroxysmal atrial fibrillation using a force-sensing catheter and 3D mapping system. CF, catheter location and orientation relative to the tissue during ablation as well as the absolute impedance decrease during the first $20 \mathrm{~s}$ of ablation as a surrogate for lesion effectiveness were analyzed for $791 \mathrm{RF}$ applications.

Results While a higher CF was achieved around the right pulmonary veins ( $12.5 v s .11 .4 \mathrm{~g}, p=0.045)$, a lower median absolute impedance decrease within the first $20 \mathrm{~s}$ was seen around the right veins compared to the left veins $(9.3 \mathrm{vs}$. $10.2 \Omega, p=0.02$ ). With different catheter orientations relative to the tissue, higher $\mathrm{CF}$ and impedance decrease was seen when the catheter was orientated parallel or oblique to the tissue $\left(30^{\circ}-145^{\circ}\right)$ as compared perpendicularly $\left(0-30^{\circ}\right)$ with a median CF of 13.2 vs. $8.0 \mathrm{~g}(p<0.001)$ and a median impedance decrease during the first $20 \mathrm{~s}$ of $11 v s .7 \Omega(p<0.001)$. Importantly, achieved CF, baseline impedance, catheter orientation and location all independently predicted the initial absolute and relative impedance decrease in a multivariable linear regression model $(p<0.05)$.
\end{abstract}

Sven Knecht and Tobias Reichlin contributed equally to this work.

Sven Knecht

sven.knecht@usb.ch

1 Department of Cardiology, University Hospital Basel, Petersgraben 4, 4031 Basel, Switzerland

2 Cardiovascular Research Institute Basel, Basel, Switzerland
Conclusions The effectiveness of RF ablation lesions, as assessed by the initial impedance decrease, is not only dependent on the achieved catheter $\mathrm{CF}$ but also on catheter orientation and location.

Keywords Atrial fibrillation · Pulmonary vein isolation · Radiofrequency ablation

\section{Introduction}

Since the first report of radiofrequency (RF) ablation in 1985 [1], several important developments in catheter technology have improved the safety and efficacy of RF energy delivery. Recently, contact force-sensing (CF) catheters (SmartTouch, Biosense Webster, Diamond Bar, USA; Tacticath, St Jude Medical, Minneapolis, USA) have been developed, enabling the operator to visualize contact information from the distal tip of the catheter. Several studies have been published, investigating the impact of contact force on acute and procedural variables [2, 3], on biophysical parameters [4-9] and on outcome after pulmonary vein isolation (PVI) [10-12]. However, contact force is only an essential but not sufficient criterion to describe the amount of energy transferred between catheter tip and the tissue. It is the amount of transferred energy that determines the heating up of the myocardium to temperatures above $50{ }^{\circ} \mathrm{C}$, causing cellular necrosis as required for radiofrequency (RF) ablation. Based on the fundamental biophysics of radiofrequency (RF) lesion formation [13], the amount of thermal energy transferred to the tissue and responsible for the subsequent temperature increase is dependent on tissue properties and the transferred electric current density. While tissue properties represent inherent variables and are potentially different between different locations in the left atrium, current density is a variable parameter roughly determined by the 
predefined power set by the generator, the contact area between the catheter tip and the tissue surface and the catheter tip design. Since tissue temperature cannot be measured directly, we use the impedance decrease as a surrogate marker for the effectiveness of RF ablation [14]. The aim of this study was to investigate the impact of the location and orientation of the catheter tip on biophysical parameters during RF ablation and on the association of contact force (CF) and impedance decrease during radiofrequency (RF) ablation using a CFsensing RF catheter.

\section{Methods}

\subsection{Study population}

We analyzed RF applications of 32 patients undergoing PVI for paroxysmal atrial fibrillation. The study complies with the Declaration of Helsinki and was approved by the local ethics committee, and informed consent was obtained from all patients.

\subsection{Electrophysiological procedure}

All subjects underwent transesophageal echocardiography to rule out left atrial thrombus the day before the procedure. Oral anticoagulation was not interrupted for the procedure in patients on vitamin $\mathrm{K}$ antagonists. In patients on dabigatran, the last dose was given $24 \mathrm{~h}$ before the procedure. All patients underwent cardiac magnetic resonance imaging or computed tomography to assess left atrial anatomy prior to the procedure. The procedures were performed with patients under conscious sedation. Double transseptal puncture was performed under fluoroscopic guidance. Intravenous heparin was used to maintain an activated clotting time of $350 \mathrm{~s}$. A 20-pole variable circumferential mapping catheter (Lasso 2515, Biosense Webster) and a $3.5-\mathrm{mm}$ open irrigated tip contact forcesensing catheter (Navistar Thermocool ST, Biosense Webster) were advanced into the left atrium. The geometrical electroanatomical reconstruction of the left atrium (Carto3, Biosense Webster) and the imported reconstruction from magnetic resonance imaging or computed tomography were used to guide the continuous circumferential antral ablation around the ipsilateral PVs. RF energy was delivered in a temperaturecontrolled mode using the EP Shuttle RF generator (Biosense Webster) with a power of $25 \mathrm{~W}(71 \%)$ or $30 \mathrm{~W}(29 \%)$ and a maximum temperature of $50{ }^{\circ} \mathrm{C}$ for a duration of $30 \mathrm{~s}$. The power was not changed during RF application and limited to $25 \mathrm{~W}$ at the posterior wall. Target contact force range was 10 $40 \mathrm{~g}$. The indifferent electrode was placed on the back of the patient at the level of the left scapula, and the irrigation fluid flow rate was set to $17 \mathrm{ml} / \mathrm{min}$.

\subsection{Data acquisition and analysis}

Point-by-point ablation was performed using the force information from the $\mathrm{CF}$-sensing catheter in conjunction with established markers of tissue contact, including tactile feedback, catheter tip motion on the 3D mapping system, electrogram quality and voltage abatement during RF energy delivery and baseline impedance and impedance decrease.

Data from ablation lesions with a minimal duration of $20 \mathrm{~s}$ and a stable contact were tagged in the system and used for analysis. Stable contact was defined by stable catheter position or uniform movement in the 3D mapping system and a uniform force pattern during the first $20 \mathrm{~s}$ of ablation. Sampling frequency was $20 \mathrm{~Hz}$ for the force measurement and $10 \mathrm{~Hz}$ for impedance. To eliminate breathing artifacts on the impedance measurements during ablation, only applications with either no respiratory influence on the impedance curve or applications acquired within the same breathing cycle at the beginning of the ablation and at $20 \mathrm{~s}$ were included for analysis.

All biophysical data (impedance, power and temperature) were post-procedurally analyzed using Matlab (Mathworks, Natick, USA). Impedance was calculated as mean over $500 \mathrm{~ms}$ at the beginning of the ablation as well as after $20 \mathrm{~s}$ to calculate the absolute and relative impedance changes within the first $20 \mathrm{~s}$. Force data were used to calculate a mean force over the first $20 \mathrm{~s}$. To assess contact force variability, we calculated the coefficient of variation $(\mathrm{CV}=\mathrm{SD} /$ mean $)$. The resulting contact force was grouped into the categories: very low $(0$ to $<5 \mathrm{~g})$, low ( 5 to $<10 \mathrm{~g})$, moderate $(10$ to $<20 \mathrm{~g}$ ) and high $(>20 \mathrm{~g})$ [11]. Temperature was calculated as mean over $500 \mathrm{~ms}$ at the beginning of the ablation, after 5 and $20 \mathrm{~s}$, and as mean temperature of the first $20 \mathrm{~s}$. For anatomical classification, the PV circumference was divided into eight segments (Fig. 1), namely the carina region, inferior circumference, left lateral ridge, "saddle" region (intersection between left lateral ridge and carina), posterior wall, roof, septum and other nonperi PV locations.

The axial angle of the resulting contact force on the catheter tip was extracted from the exported data set. This CF vector orientation was used as quantitative measure for the catheter orientation relative to the tissue surface. An angle of $0^{\circ}$ represents a perpendicular orientation of the catheter tip to the endocardial surface whereas $90^{\circ}$ describes a parallel catheter orientation to the surface. The catheter orientation was classified into three groups, namely perpendicular catheter orientation ( 0 to $<30^{\circ}$ : $0^{\circ}$ group), transverse catheter orientation $\left(30^{\circ}\right.$ to $<60^{\circ}: 45^{\circ}$ group) and parallel catheter orientation $\left(60^{\circ}\right.$ to $<150^{\circ}$ : $90^{\circ}$ group) (Fig. 2).

\subsection{Statistical analysis}

Continuous variables are presented as mean \pm standard deviation (SD) or median with interquartile range (IQR). 
Fig. 1 Visualization of the anatomical classification into eight segments for the left pulmonary veins (a) and right pulmonary veins (b) (a)

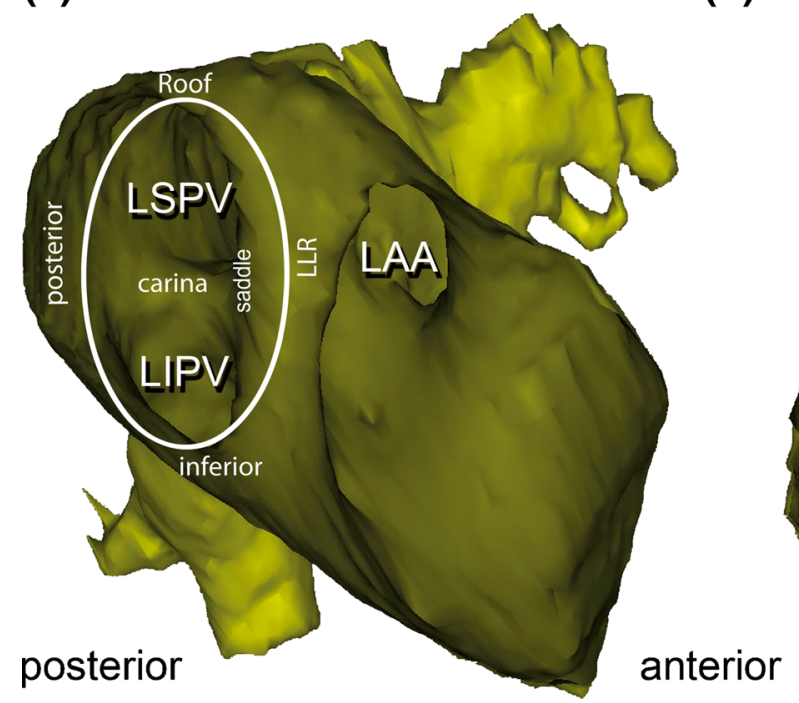

(b)

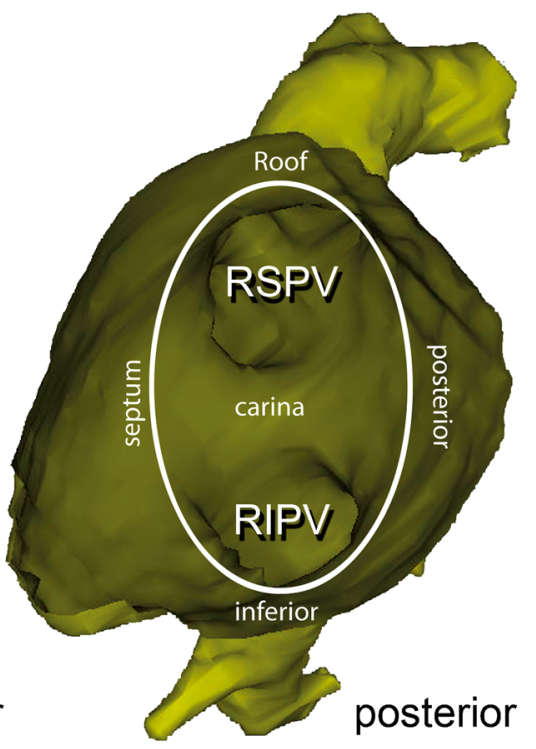

Categorical variables are presented as numbers and percentages. Continuous variables were compared with the MannWhitney $U$ test and categorical variables using the Pearson chi-square test. A one-way ANOVA with a Tukey's post hoc test was used for comparisons between multiple groups. Correlation was tested using the Pearson's product moment correlation coefficient and the Spearman's rank correlation coefficient as adequate. A stepwise multivariable linear regression model was used to calculate the relationship between the biophysical parameters on the impedance that decrease as a surrogate for the effectiveness of ablation. Analysis was performed using SPSS (IBM SPSS Statistics for Windows, Version 22.0. Armonk, NY, USA). A $p<0.05$ was considered statistically significant.

\section{Results}

A total of 791 lesions fulfilling the above described stability criteria over the first $20 \mathrm{~s}$ of ablation were analyzed from 32 patients. The patients had a median age of 58 years, median weight and height of $82 \mathrm{~kg}$ and $176 \mathrm{~cm}$, a median left atrial size of $42 \mathrm{~mm}$ and a left ventricular ejection fraction of $57 \%$.

\subsection{Biophysical parameters}

Power was set to $25 \mathrm{~W}$ for 560 applications (71\%) and to $30 \mathrm{~W}$ for 231 applications (29\%). The power was not significantly different between locations and angulation. The median baseline impedance was $126 \Omega$ (119-134), and the median absolute and relative impedance decreases after $20 \mathrm{~s}$ were 10 $\Omega(6-15)$ and $8 \%(5-11)$, respectively. Mean temperature at baseline, after 5 and $20 \mathrm{~s}$, was $33{ }^{\circ} \mathrm{C}(32-33), 37{ }^{\circ} \mathrm{C}$ (36-38) and $38{ }^{\circ} \mathrm{C}(37-40)$ respectively. The median of the achieved CF was $12.0 \mathrm{~g}$ (7.9-17.5). Very low $(<5 \mathrm{~g})$ and high force $(>20 \mathrm{~g})$ were observed in $76(10 \%)$ and $133(17 \%)$ of cases. The majority of ablations were performed with low $(229,29 \%)$ and moderate (353, $45 \%) \mathrm{CF}$. The median coefficient of variation of the CF was $0.36(0.25-0.49)$.
Fig. 2 Visualization of the angle orientation of the contact force in relation to the catheter tip of the three groups
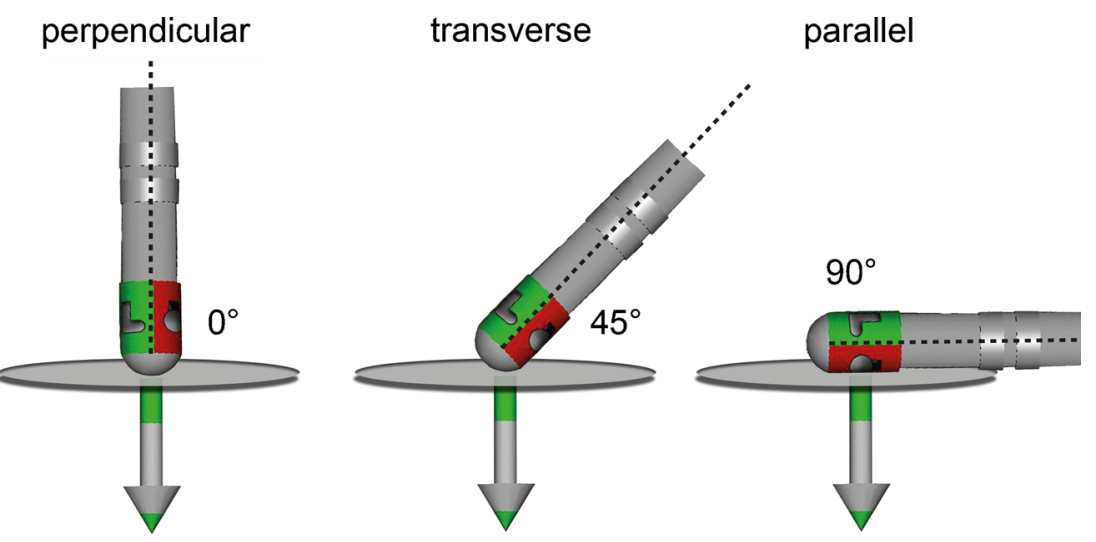


\subsection{Location dependency}

Based on anatomical location, $416 \mathrm{RF}$ applications were performed around the left PVs, 354 around the right PVs and 21 applications in the atrial tissue in non-peri PV locations. More specifically, 79 ablations were performed in the carina region (left and right), 122 at the left lateral ridge, 84 and 76 at the roof and inferior circumference around the PV, 67 in the saddle region, 247 at the posterior wall and 100 at the septum, respectively.

Between the left and the right PVs, significant differences could be found for the achieved $\mathrm{CF}$, the coefficient of variation of the $\mathrm{CF}$, the absolute and relative impedance decrease during ablation, the temperatures after 5 and $20 \mathrm{~s}$ and the mean temperature over the $20 \mathrm{~s}$ (Table 1). There was a significant difference between the anatomical location and all biophysical parameters, except for the coefficient of variation of the $\mathrm{CF}$ (Fig. 3). CF was significantly higher at the roof and the posterior wall than at the carina, the left lateral ridge, the saddle region and in the LA.

\subsection{Catheter orientation}

Mean catheter orientation for all ablations was $50^{\circ} \pm 34$. Based on anatomical locations, the median catheter orientation was $51^{\circ}(26-81)$ for the carina region, $48^{\circ}(25-71)$ for the left lateral ridge, $53^{\circ}(30-75)$ degree for the roof, $48^{\circ}(32-65)$ for the inferior area around the $\mathrm{PV}, 53^{\circ}(35-75)$ for the saddle region, $38^{\circ}(20-59)$ for the posterior wall and $63^{\circ}(41-80)$ for the septum $(p<0.001)$ (Fig. 3). The catheter orientation at the posterior wall was significantly different from the angle at the roof $(p=0.007)$ and the septum $(p<0.000)$ with a more perpendicular catheter orientation at the posterior wall. Mean values of the catheter orientation, the contact force and the resulting impedance decrease is shown for every segment in Fig. 4.
Fig. 3 Boxplots of the biophysical parameters for every segment. $L L R$ left lateral ridge, $L A$ left atrium; ${ }^{\#} p<0.05$

There was a significant difference in the $\mathrm{CF}$, the coefficient of variation for the $\mathrm{CF}$, the absolute and relative impedance decrease during ablation and all temperatures for the three groups of catheter orientation, but not for power and baseline impedance (Table 2). The variance of the force is significantly different between the angulations of the groups and highest for perpendicular catheter orientations. With a parallel or transverse catheter orientation $\left(30-145^{\circ}\right)$ as compared to a perpendicular orientation $\left(0-30^{\circ}\right)$, a higher median $\mathrm{CF}$ of $13.2 \mathrm{vs}$. $7.9 \mathrm{~g}(p<0.001)$, and a median impedance decrease during the first $20 \mathrm{~s}$ of $11 v s .7 \Omega(p<0.001)$ could be observed.

\subsection{Interdependence of parameters}

\subsubsection{Bivariate association of biophysical parameters}

A significant correlation was found between the initial absolute and relative impedance decrease and the mean contact force $(R=0.400$ and $0.416, p<0.001)$ and the coefficient of variation of CF $(R=-0.236$ and $-0.244, p<0.001)$. For the absolute and relative impedance decrease, we found a significant correlation with the baseline impedance $(R=0.531$ and $0.403, p<0.001)$, the temperature at $5 \mathrm{~s}(R=0.329$ and $R=$ $0.312, p<0.001)$ and the temperature at $20 \mathrm{~s}(R=0.337$ and $0.329, p<0.001)$. There is a linear correlation between the coefficient of variance and the force $(R=-0.251, p<0.001)$.

\subsubsection{Impact of catheter orientation and location on the association of $C F$ and absolute impedance decrease}

Table 3 shows the association of $\mathrm{CF}$ and resulting absolute impedance decrease in relation to anatomical location and
Table 1 Differences of the biophysical data between left and right-sided pulmonary veins

\begin{tabular}{lllr}
\hline Parameter & $\begin{array}{l}\text { Left pulmonary vein } \\
(n=416)\end{array}$ & $\begin{array}{l}\text { Right pulmonary vein } \\
(n=354)\end{array}$ & $P$ value \\
\hline Contact force $[\mathrm{g}]$ & $11.4(7.6-17.3)$ & $12.5(8.3-18.0)$ & 0.045 \\
Contact force COV [-] & $0.33(0.23-0.44)$ & $0.39(0.28-0.52)$ & $<0.001$ \\
ImpStart $[\Omega]$ & $126(135-119)$ & $125(119-133)$ & 0.232 \\
absDeltaImp $[\Omega]$ & $10.2(6.5-14.7)$ & $9.3(5.2-14.3)$ & 0.016 \\
relDeltaImp [\%] & $8.1(5.3-11.5)$ & $7.5(4.3-11.1)$ & 0.018 \\
Tstart & $32.0(32.0-33.0)$ & $33.0(32.0-33.0)$ & $<0.001$ \\
$\mathrm{~T}_{5 \mathrm{~s}}\left[{ }^{\circ} \mathrm{C}\right]$ & $37.0(36.0-37.9)$ & $37.0(36.0-38.0)$ & 0.049 \\
$\mathrm{~T}_{20 \mathrm{~s}}\left[{ }^{\circ} \mathrm{C}\right]$ & $38.0(37.0-39.7)$ & $38.2(37.0-40.0)$ & 0.072 \\
$\mathrm{~T}_{\text {mean }}\left[{ }^{\circ} \mathrm{C}\right]$ & $37.2(36.3-38.5)$ & $37.5(36.6-38.6)$ & 0.023 \\
Power $[$ watts] & $25(25-29)$ & $25(25-29)$ & 0.726 \\
\hline
\end{tabular}

All values are given as median (IQR)

COV coefficient of variation, ImpStart baseline impedance, absDeltaImp absolute impedance decrease, relDeltaImp relative impedance decrease, $T$ temperature 

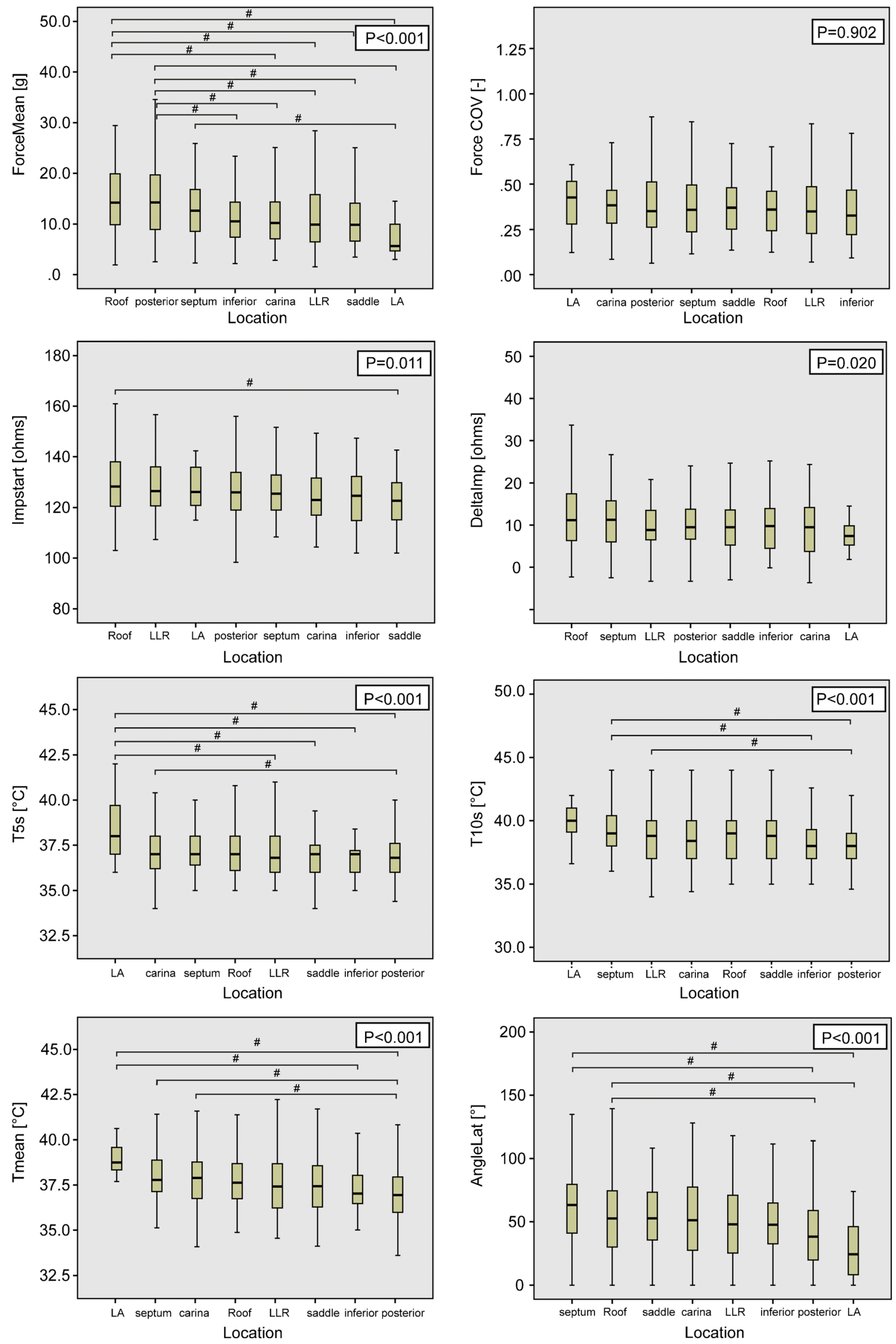


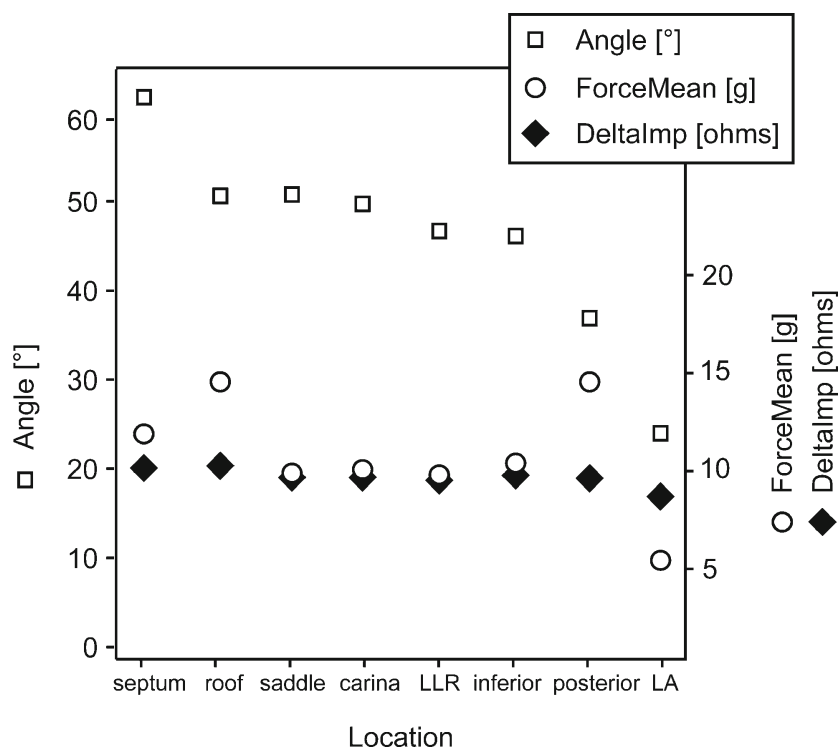

Fig. 4 Mean values of the lateral angle (square), the contact force (circle) and the resulting impedance decrease for every segment

catheter orientation. With similar achieved CF, higher impedance decreases were seen during ablation at the LPVs compared to the RPV, but no association was found between segmental locations and the 4 groups of CF. With regard to the different catheter orientations, higher impedance decreases were seen during ablation with similar CF achieved when the catheter was orientated parallel or oblique to the tissue $\left(30-145^{\circ}\right)$ as compared to perpendicular $\left(0-30^{\circ}\right)$.

\subsection{Multiple linear regression}

The achieved CF, baseline impedance, catheter orientation and ablation at the left PVs and the septum were significantly associated with the absolute impedance decrease in our multiple linear regression model $\left(R^{2}=0.44, R=0.66, p<0.05\right.$ for all predictors) (Table 4).

Relative impedance decrease was significantly associated with the achieved $\mathrm{CF}$, the baseline impedance, the catheter orientation and the ablations at the left PVs and the septum $\left(R^{2}=0.35, R=0.59, p<0.05\right.$ for all included predictors).

\section{Discussion}

In this study, we could show for the first time that the anatomic location and orientation of the catheter tip during RF ablation have an important impact on the biophysical parameters measured during ablation, most importantly the association between achieved $\mathrm{CF}$ and the resulting initial impedance decrease during ablation. We identified the baseline impedance, the achieved CF, the catheter orientation and location as independent predictors for the effectiveness of RF ablation with the initial impedance decrease used as a surrogate measure.

With the availability of CF measurements from RF ablation catheters, numerous studies have been published focusing on the effect of $\mathrm{CF}$ on the efficacy of radiofrequency energy delivery [4-9]. CF has the potential to tell the operator that he will deliver a lesion before any RF energy is actually delivered. From a biophysical point of view, however, CF is a necessary but not sufficient criterion to predict energy transfer to the tissue. As tissue temperature cannot be measured, biophysical parameters such as impedance decrease, reflecting indirectly tissue heating $[14,15]$, or electrogram criteria, reflecting the transmurality of the lesions [16], are currently used as surrogates for lesion formation. Recently, associations between contact force and initial impedance decrease could be shown $[6,8,17]$. However, differences in location and
Table 2 Difference of the biophysical data between the 4 groups of catheter orientation

\begin{tabular}{llllr}
\hline Parameter & $\begin{array}{l}0^{\circ} \text { group } \\
(n=232)\end{array}$ & $\begin{array}{l}45^{\circ} \text { group } \\
(n=283)\end{array}$ & $\begin{array}{l}90^{\circ} \text { group } \\
(n=276)\end{array}$ & $P$ value \\
\hline Contact force $[\mathrm{g}]$ & $7.9(4.7-14.2)$ & $13.0(8.8-18.8)$ & $13.4(10.2-18.1)$ & $<0.001$ \\
Force COV $[-]$ & $0.49(0.36-0.65)$ & $0.32(0.22-0.41)$ & $0.31(0.21-0.42)$ & $<0.001$ \\
ImpStart $[\Omega]$ & $124(119-133)$ & $126(118-134)$ & $126(117-134)$ & 0.625 \\
absDeltaImp $[\Omega]$ & $6.8(3.4-10.3)$ & $10.3(7.0-14.7)$ & $11.8(7.8-16.5)$ & $<0.001$ \\
relDeltaImp $[\%]$ & $5.5(2.8-8.2)$ & $8.2(5.6-11.4)$ & $9.7(6.3-12.9)$ & $<0.001$ \\
Tstart $\left[{ }^{\circ} \mathrm{C}\right]$ & $33.0(32.0-33.0)$ & $32.0(32.0-33.0)^{*}$ & $32.0(32.0-33.0)^{*}$ & $<0.001$ \\
$\mathrm{~T}_{5 \mathrm{~s}}\left[{ }^{\circ} \mathrm{C}\right]$ & $36.8(36.0-37.2)$ & $37.0(36.0-38.0)$ & $37.0(36.4-38.0)^{*}$ & $<0.001$ \\
$\mathrm{~T}_{20 \mathrm{~s}}\left[{ }^{\circ} \mathrm{C}\right]$ & $38.0(37.0-39.0)$ & $38.0(37.0-40.0)$ & $39.0(37.3-40.3)$ & $<0.001$ \\
$\mathrm{~T}_{\text {mean }}\left[{ }^{\circ} \mathrm{C}\right]$ & $37.0(36.1-38.0)$ & $37.4(36.5-38.5)$ & $37.7(36.8-39.3)^{*}, \#$ & $<0.001$ \\
Power $[\mathrm{W}]$ & $25(25-30)$ & $25(25-29)$ & $25(25-29)$ & 0.864 \\
\hline
\end{tabular}

All values are given as median (IQR)

COV coefficient of variation, ImpStart baseline impedance, absDeltaImp absolute impedance decrease, relDeltaImp relative impedance decrease, $T$ temperature

${ }^{*} p<0.05$ (significant difference to $0^{\circ}$ group); ${ }^{*} p<0.05$ (significant difference to $90^{\circ}$ group) 
Table 3 Summary of the absolute impedance decrease in ohms for all 4 force groups for the anatomical conditions

\begin{tabular}{|c|c|c|c|c|}
\hline & $\begin{array}{l}\text { Very low } \\
(0-5 \mathrm{~g}) \\
(n=76)\end{array}$ & $\begin{array}{l}\text { Low } \\
(5-10 \mathrm{~g}) \\
(n=229)\end{array}$ & $\begin{array}{l}\text { Moderate } \\
(10-20 \mathrm{~g}) \\
(n=353)\end{array}$ & $\begin{array}{l}\text { High } \\
(>20 \mathrm{~g}) \\
(n=133)\end{array}$ \\
\hline \multicolumn{5}{|c|}{ Overall reference } \\
\hline absDeltaImp & $4.9(2.2-7.5)$ & $8.0(4.4-11.2)$ & $10.5(7.3-15.8)$ & $13.7(10-18.8)$ \\
\hline \multicolumn{5}{|l|}{ Atrial aspect } \\
\hline LPV & $4.9(2.3-7.8)$ & $8.7(5.8-11.8)$ & $11.7(8.3-16.3)$ & $15.0(10.0-20.8)$ \\
\hline RPV & $4.8(1.9-7.0)$ & $6.7(2.7-10.3)$ & $9.7(6.8-15.4)$ & $13.2(10.0-17)$ \\
\hline$P$ value & 0.772 & 0.004 & 0.018 & 0.175 \\
\hline \multicolumn{5}{|l|}{ Angle } \\
\hline $0^{\circ}$ group & $4.5(1.9-7.3)$ & $6.5(3.3-9.0)^{\mathrm{a}}$ & $8.7(5.4-11.7)^{\mathrm{a}}$ & $10.8(7.0-13.4)^{\mathrm{a}}$ \\
\hline $45^{\circ}$ group & $6.5(0.5-8.4)$ & $8.5(4.0-11.7)$ & $10.3(7.7-14.9)$ & $14.7(11.2-19.1)$ \\
\hline $90^{\circ}$ group & $6.1(2.9-7.9)$ & $10.3(6.7-13.5)^{\mathrm{a}}$ & $12.3(7.8-16.7)^{\mathrm{a}}$ & $14.7(9.5-20.8)^{\mathrm{a}}$ \\
\hline$P$ value & 0.989 & 0.002 & 0.000 & 0.029 \\
\hline \multicolumn{5}{|l|}{ Location } \\
\hline Carina & $2.5(0-11.9)$ & $7.8(2.8-11.3)$ & $11.8(7.6-16.3)$ & $12.6(11.0-17.8)$ \\
\hline Inferior & $2.1(0.8-6.0)$ & $5.8(2.9-11.1)$ & $11.1(7.5-15.4)$ & $12.6(11.0-21.0)$ \\
\hline LLR & $6.2(3.1-7.7)$ & $8.5(6.8-10.9)$ & $11.3(7.9-16.9)$ & $13.3(7.7-20.8)$ \\
\hline Saddle & $3.8(-1.2-7.0)$ & $8.8(4.1-13.5)$ & $12.2(7.2-14.8)$ & $11.8(9.6-16.0)$ \\
\hline Posterior & $7.0(2.2-7.3)$ & $7.5(3.8-10.6)$ & $9.8(7.3-14.3)$ & $12.6(8.5-17.8)$ \\
\hline Roof & $6.7(0.8-11.0)$ & $6.3(1.3-11.2)$ & $11.7(7.2-18.8)$ & $16.0(12.1-25.0)$ \\
\hline Septum & $5.2(2.8-8.4)$ & $11.0(5.4-14.1)$ & $11.0(6.6-16.0)$ & $15.7(13.3-20.3)$ \\
\hline LA & $6.6(5.6-8.8)$ & $7.4(2.6-9.8)$ & $10.3(6.2-15.7)$ & $\mathrm{n} / \mathrm{a}$ \\
\hline$P$ value & 0.825 & 0.248 & 0.449 & 0.247 \\
\hline
\end{tabular}

All values in ohms $[\Omega]$ are given as median (IQR)

$L L R$ left lateral ridge, $L P V$ left pulmonary veins, $R P V$ right pulmonary veins, $n / a$ not available

${ }^{a}$ Significant difference from Tukey post hoc analysis
Table 4 Multiple linear regression model for the absolute and relative impedance decrease

\begin{tabular}{ccccc}
\hline Variable & Predictors & $\begin{array}{l}\text { Non-standardized } \\
\text { coefficient }\end{array}$ & $\begin{array}{l}\text { Standardized } \\
\text { coefficient }\end{array}$ & $P$ value \\
\hline absDeltaImp & & & \\
Constant & -32.55 & & $<0.001$ \\
ImpStart & 0.291 & 0.481 & $<0.001$ \\
ForceMean & 0.266 & 0.284 & $<0.001$ \\
AngleGroup & 0.037 & 0.189 & $<0.001$ \\
LPV & 1.443 & 0.103 & $<0.001$ \\
Septum & 1.162 & 0.064 & 0.012 \\
relDeltaImp & & & \\
Constant & -0.157 & & $<0.001$ \\
ForceMean & 0.002 & 0.316 & $<0.001$ \\
ImpStart & 0.001 & 0.348 & $<0.001$ \\
AngleGroup & 0.000 & 0.209 & $<0.001$ \\
LPV & 0.011 & 0.114 & 0.001 \\
Septum & 0.009 & 0.073 & 0.037 \\
\hline
\end{tabular}

absDeltaImp absolute impedance decrease, ImpStart baseline impedance, $L P V$ left pulmonary veins quantitative catheter orientation were not assessed and included in those analyses. Our results confirm and corroborate findings from a previous small pilot study that has investigated the role of catheter orientation using a semi-quantitative catheter orientation score and reported an association of catheter orientation and impedance decrease when delivering RF lesions in healthy tissue with a local electrogram amplitude $>2 \mathrm{mV}$ [5].

\subsection{Biophysical aspects of RF ablation}

As shown by Gallagher et al. [18], the electrode tissue contact is geometrically determined by two aspects: (1) the penetration depth of the catheter tip into the tissue and (2) the catheter orientation. Both parameters determine the contact area and, consequently, the amount of energy transfer, resulting in the targeted heating of the tissue. Contact force measurements, however, do not only reflect the resulting reactive force of the myocardial tissue due to its deformation but as well as the reactive forces of the surrounding structures such as the ascending and descending Aorta, as shown by Nakagawa et al. [19]. High forces in this area reflect the "bearing" of this 
structure, allowing only slight deformation of the myocardial tissue and preventing the "embedding" of the catheter tip in the tissue, which would result in greater contact area and, consequently, a greater energy transfer to the tissue. Differences of these surrounding anatomical conditions between patients might consequently explain variations in impedance decrease. Furthermore, baseline impedance differences could be identified between the left atrial and the pulmonary vein ostial tissue, with higher impedance around and within the pulmonary veins [20].

Catheter tip temperature has shown its potential to predict lesion formation [21]. With the introduction of the irrigated tip catheters and especially with the recently launched highly effectively cooled "porous" catheters, however, this information is lost despite being the only direct measurement of the effective energy transfer for catheter ablation. With the six holeirrigated tip catheters used in this study, however, statistical differences between the anatomical locations and a moderate correlation between the impedance decrease and temperatures could be identified. Furthermore, temperatures reached during ablation were significantly higher in areas with a parallel contact (e.g. the septum) than with a perpendicular contact (e.g. posterior and inferior areas), showing the potential of temperature measurements for improved assessment of lesion formation. However, whether new technical developments such as optimized positioning of temperature electrodes on the catheter tip result in better predictive models for the efficacy of catheter ablation has to be investigated.

\subsection{Anatomical location}

Contact force analysis during left atrial mapping with the operator blinded to the force value identified a higher variability of CF $[9,19]$ than observed in our non-blinded study. One reason for this may be our predefined maximal $\mathrm{CF}$ of $40 \mathrm{~g}$ to prevent perforation and tamponade. However, as in other studies, including detailed location information, we observed a significantly higher mean contact force for the ablations around the right pulmonary veins $[5,9,12]$. It is an important finding of our study that despite higher CF achieved at the right pulmonary veins, the resulting initial impedance decreases as a marker of lesion formation was greater with ablation at the left veins. This underscores the notion that $\mathrm{CF}$ is a necessary but not the sole factor that determines the effectiveness of a resulting lesion and the hypothesis that the myocardial tissue characteristics have a significant impact on the association of $\mathrm{CF}$ and resulting impedance decrease. Furthermore, differences in fluid flow around the catheter tip, which are as well dependent on its location, have an impact on the heat transfer to the tissue and might explain the differences in impedance drop with location independent on the force.

\subsection{Catheter orientation relative to the tissue}

Computational simulations have shown that catheter orientation has a large effect on lesion volume [18]. A more parallel catheter orientation but the same penetration depth resulted in lesion volume increase of up to $70 \%$ due to the greater contact area and, consequently, more energy transfer to the tissue. With higher CF, however, this effect may be reduced because the contact area is more determined by the penetration depth of the catheter tip. In our study, for RF ablations with a similar $\mathrm{CF}$, an increase of the contact orientation from perpendicular $\left(0^{\circ}\right)$ to transverse $\left(45^{\circ}\right)$ or even parallel $\left(90^{\circ}\right)$ resulted in an increase of the absolute initial impedance drop by $20-40$ and $35-60 \%$, respectively. This is in line with the above simulation-based hypothesis that the same CF results in a larger lesion with a more parallel catheter orientation.

A high coefficient of variation does not inevitably mean unstable catheter contact but, by definition, a high variation in force relative to the mean achieved $\mathrm{CF}$. This can reflect tissue or locations with more contraction or the flexibility or stiffness of the catheter. As shown in our study, sites with a more parallel orientation show a lower variation in CF than sites with perpendicular contact since the catheter with parallel orientation can follow the wall movements better. With the same maximal force limited due to safety concerns, a more parallel catheter orientation with more constant force may translate into larger lesions [22].

In numerical simulations of RF catheter ablations, lesion volume with a temperature of more than $50{ }^{\circ} \mathrm{C}$ was significantly higher for almost parallel catheter orientation $\left(75^{\circ}\right)$ compared to perpendicular orientation, with the same penetration depth of the catheter within the tissue [18].

\subsection{Clinical implication}

We confirm previous studies indicating that catheter $\mathrm{CF}$ is an important factor for effective lesion generation. Furthermore, our study indicates that the anatomic location and catheter orientation are independently predictive as well. Especially in areas with low achieved $\mathrm{CF}$, attempting to achieve a more parallel catheter orientation may be an option to increase the energy transfer and ultimately generate effective RF lesions.

\section{Limitations}

This is a non-randomized single-centre study with a limited number of patients but a high number of individually analysed lesions. In this clinical study and in contrast to studies in the animal lab, no direct visual and histological assessment of the resulting lesions is possible. Furthermore, changes in tissue temperature cannot be reliably measured with irrigated catheters. The initial impedance decrease was therefore used as a 
surrogate for the effectiveness of catheter ablation. The results were derived from the analysis of ablations within the left atrium in areas with thin, flat myocardial tissue. Whether and how this can be transferred to thicker, pectinate muscular structure such as in the ventricles, the right atrium or the appendages is unclear.

\section{Conclusion}

The effectiveness of RF ablation lesions, as assessed by the initial impedance decrease, is not only dependent on the achieved catheter CF but importantly also on catheter orientation and location. With similar achieved catheter CF, greater impedance decreases were observed around the left versus the right pulmonary veins and with a more parallel compared to a perpendicular catheter orientation.

Conflict of interest Sven Knecht, Tobias Reichlin and Beat Schaer have no conflict of interest. Nikola Pavlovic is supported by an educational grant of the EHRA. Stefan Osswald is a speaker of Bureau for Medtronic, Boston Scientific, Biotronik and St. Jude Medical received unrestricted grants from Medtronic, Boston Scientific, Biotronik and St. Jude Medical. Christian Sticherling is a member of Medtronic Advisory Board Europe and received educational grants from Biosense Webster and Biotronik. Michael Kühne received educational grants from Biosense Webster, proctor for Medtronic and a speaker of Bureau for Boston Scientific, St. Jude Medical and Biotronik.

\section{References}

1. Huang, S. K., Bharati, S., Graham, A. R., Lev, M., Marcus, F. I., \& Odell, R. C. (1987). Closed chest catheter desiccation of the atrioventricular junction using radiofrequency energy - a new method of catheter ablation. Journal of the American College of Cardiology, 9, 349-358.

2. Haldar, S., Jarman, J. W. E., Panikker, S., Jones, D. G., Salukhe, T., Gupta, D., et al. (2013). Contact force sensing technology identifies sites of inadequate contact and reduces acute pulmonary vein reconnection: a prospective case control study. International Journal of Cardiology, 168, 1160-1166.

3. Stabile, G., Solimene, F., Calò, L., Anselmino, M., Castro, A., Pratola, C., et al. (2014). Catheter-tissue contact force for pulmonary veins isolation: a pilot multicentre study on effect on procedure and fluoroscopy time. Europace, 16, 335-340.

4. Bortone, A., Appetiti, A., Bouzeman, A., Maupas, E., Ciobotaru, V., Boulenc, J.-M., et al. (2013). Unipolar signal modification as a guide for lesion creation during radiofrequency application in the left atrium: prospective study in humans in the setting of paroxysmal atrial fibrillation catheter ablation. Circulation. Arrhythmia and Electrophysiology, 6, 1095-1102.

5. De Bortoli, A., Sun, L.-Z., Solheim, E., Hoff, P. I., Schuster, P., Ohm, O.-J., et al. (2013). Ablation effect indicated by impedance fall is correlated with contact force level during ablation for atrial fibrillation. Journal of Cardiovascular Electrophysiology, 24, 1210-1215.

6. Reichlin, T., Knecht, S., Lane, C., Kühne, M., Nof, E., Chopra, N., et al. (2014). Initial impedance decrease as an indicator of good catheter contact: Insights from radiofrequency ablation with force sensing catheters. Heart Rhythm, 11, 194-201.

7. Kumar, S., Chan, M., Lee, J., Wong, M. C. G., Yudi, M., Morton, J. B., et al. (2014). Catheter-tissue contact force determines atrial electrogram characteristics before and lesion efficacy after antral pulmonary vein isolation in humans. Journal of Cardiovascular Electrophysiology, 25, 122-129.

8. Kumar, S., Haqqani, H. M., Chan, M., Lee, J., Yudi, M., Wong, M. C. G., et al. (2013). Predictive value of impedance changes for realtime contact force measurements during catheter ablation of atrial arrhythmias in humans. Heart Rhythm, 10, 962-969.

9. Makimoto, H., Metzner, A., Lin, T., Rillig, A., Wohlmuth, P., Arya, A., et al. (2014). In vivo contact force analysis and correlation with tissue impedance during left atrial mapping and catheter ablation of atrial fibrillation. Circulation. Arrhythmia and Electrophysiology, 7, 46-54.

10. Neuzil, P., Reddy, V. Y., Kautzner, J., Petru, J., Wichterle, D., Shah, D., et al. (2013). Electrical reconnection after pulmonary vein isolation is contingent on contact force during initial treatment: results from the EFFICAS I study. Circulation. Arrhythmia and Electrophysiology, 6, 327-333.

11. Reddy, V. Y., Shah, D., Kautzner, J., Schmidt, B., Saoudi, N., Herrera, C., et al. (2012). The relationship between contact force and clinical outcome during radiofrequency catheter ablation of atrial fibrillation in the TOCCATA study. Heart Rhythm, 9, 17891795.

12. Kumar, S., Morton, J. B., Lee, J., Halloran, K., Spence, S. J., Gorelik, A., et al. (2012). Prospective characterization of cathetertissue contact force at different anatomic sites during antral pulmonary vein isolation. Circulation. Arrhythmia and Electrophysiology, $5,1124-1129$.

13. Tungjitkusolmun, S., Woo, E. J., Cao, H., Tsai, J. Z., Vorperian, V. R., \& Webster, P. J. G. (2000). Thermal-electrical finite element modelling for radio frequency cardiac ablation: effects of changes in myocardial properties. Medical and Biological Engineering and Computing, 38, 562-568.

14. Haines, D. (2011). Biophysics of radiofrequency lesion formation. In S. K. S. Huang \& M. A. Wood (Ed.), Catheter ablation of cardiac arrhythmias, Second edition (pp 2-19). Philadelphia, PA: Elsevier Saunders.

15. Bosnos, M., Guillen-Rodriguez, J. M., He, D. S., \& Marcus, F. I. (2010). Early assessment of biophysical parameters predicts lesion formation during RF energy delivery in vitro. Pace, 33, 1082-1088.

16. Otomo, K., Uno, K., Fujiwara, H., Isobe, M., \& Iesaka, Y. (2010). Local unipolar and bipolar electrogram criteria for evaluating the transmurality of atrial ablation lesions at different catheter orientations relative to the endocardial surface. Heart Rhythm, 7, 1291-1300.

17. Ullah, W., Hunter, R. J., Baker, V., Dhinoja, M. B., Sporton, S., Earley, M., et al. (2014). Target indices for clinical ablation in atrial fibrillation: insights from contact force, electrogram and biophysical parameter analysis. Circulation. Arrhythmia and Electrophysiology, 7, 63-68.

18. Gallagher, N., Fear, E. C., Byrd, I. A., \& Vigmond, E. J. (2013). Contact geometry affects lesion formation in radio-frequency cardiac catheter ablation. PLoS One, 8, e73242.

19. Nakagawa, H., Kautzner, J., Natale, A., Peichl, P., Cihak, R., Wichterle, D., et al. (2013). Locations of high contact force during left atrial mapping in atrial fibrillation patients: electrogram amplitude and impedance are poor predictors of electrode-tissue contact force for ablation of atrial fibrillation. Circulation. Arrhythmia and Electrophysiology, 6, 746-753.

20. Lang, C. C. E., Gugliotta, F., Santinelli, V., Mesas, C., Tomita, T., Vicedomini, G., et al. (2006). Endocardial impedance mapping during circumferential pulmonary vein ablation of atrial fibrillation differentiates between atrial and venous tissue. Heart Rhythm, 3 , $171-178$. 
21. Høgh Petersen, H., Chen, X., Pietersen, A., Svendsen, J. H., \& Haunsø, S. (1999). Lesion dimensions during temperaturecontrolled radiofrequency catheter ablation of left ventricular porcine myocardium: impact of ablation site, electrode size, and convective cooling. Circulation, 99, 319-325.
22. Shah, D. C., Lambert, H., Nakagawa, H., Langenkamp, A., Aeby, N., \& Leo, G. (2010). Area under the real-time contact force curve predicts radiofrequency lesion size in an in vitro contractile model. Journal of Cardiovascular Electrophysiology, 21, 1038-1043. 